\title{
Numerical Analysis of a Wind Turbine Blade with Different Software
}

\author{
Gorazd HREN
}

\begin{abstract}
The development of wind power generation technology recognises the wind turbine blade design and manufacturing as crucial for its performance. The laboratory size wind turbine blade was analysed, considering the 3D printing plastic material for blades. Applying the structural dynamic equations of blades, the aero-elastic model of the rotating rotor and the numerical simulation method of the deflections are presented under the aerodynamic loads and centrifugal forces. Based on the parameters of aerofoil and the geometrical parameters of blade, 3D model of the blade was established with the modelling software. Then the model was introduced into computational flow dynamics software to carry out the loads on the blade and further into numerical structural analysis. The analysis was performed in two different software packages. Design of wind turbine blades depends on high precision, reliable and robust numerical predictions of performance where the plug-in software is found to be inadequate for nontrivial problems.
\end{abstract}

Keywords: computational fluid dynamics; numerical analysis; structural analysis; wind turbine blade

\section{INTRODUCTION}

One of the major challenges in this century is the efficient use of energy resources, as well as the growing production of energy from renewable sources. There are several, developed and developing, alternative forms of energy that have been explored, including geothermal, solar, wind and hydroelectric power. The affordability and performance of renewable energy technologies is the key to ensure its availability to the market.

A wind turbine uses the aerodynamic force of lift to rotate a shaft, which in turn aids in the conversion of mechanical power to electricity by means of a generator. For large networks, modern wind turbines are connected to the grid in a wind farm and serve the purpose of reducing the total electrical load. One significant downside of wind as a resource is its variable speed and fluctuating electrical output. The most common design of a wind turbine is the horizontal axis wind turbine (HAWT) [1].

The rotor consists of a hub and multiple wind turbine blades. These components are often considered the most important for cost efficiency. To achieve the maximum power from a given wind situation, the geometry of the blade is designed to provide the maximum power coefficient at a given wind speed and angular velocity. Numerical solutions based on analytical equations are adequate tools for the design of a wind turbine blade in order to get maximum power from a given wind [2].

Wind turbine blades conceptual design is inherently a multi-disciplinary design process that involves numerous disciplines and forms of expertise. In this paper, how Computer-Aided Engineering (CAE) software can be used to accelerate the creation and preparation of wind rotor blades geometry for Computational Fluid Dynamics (CFD) analysis is investigated. Nowadays, Blade Element Momentum theory (BEM) [3] and CFD analysis are base methods to design and analyse a wind turbine blade. CFD code simulations are accurate but very time consuming. BEM based codes are faster but they are not able to calculate the local stress/strain that appear at different positions on the blade. This paper presents the development of a laboratory size wind turbine blade and aero-structural analysis that calculates the global and local stress/strain results on a wind turbine blade for optimal wind conditions.

\section{PARAMETERS AND DESIGN OF THE WIND TURBINE BLADE}

We designed the wind turbine blade for laboratory testing with a diameter of $0.9 \mathrm{~m}$ and hub diameter of $0.09 \mathrm{~m}$. The designed blade used the aerofoil profile NREL S826. It is a profile with special characteristics and very pronounced curves that are hard to manufacture, but suitable for high quality $3 \mathrm{D}$ printing. The size of the blade is determined with available volumes of the $3 \mathrm{D}$ printing and the wind tunnel for measuring.

NRELS826 aerodynamic profile was designed at the National Renewable Energy Laboratory (NREL) in the USA for variable speeds and a Reynolds number of $2.0 \times 10^{6}$, which is higher than it is expected for the wind turbine. The design of the aerofoil is made in the way that the aerofoil should be less sensitive to leading edge roughness, by putting a highly curved part on the suction side, close to the leading edge. In the range of expected Reynolds numbers this suction peak causes laminar instability, but due to the lower Reynolds number as it is designed for, the flow remains laminar over a larger portion of the aerofoil [4]. The aerofoil is also equipped with a separation ramp near the trailing edge which increases the adverse pressure gradient and leads to flow separation. When the flow separates, the separation tends to be confined to the separation area, reducing the dynamic stall effects. The aerofoil is cambered, and therefore generates lift at zero angle of attack [4].

To characterise an aerofoil a number of terms are used. The most forward and rearward points of the mean camber line are on the leading edge and trailing edge. The line connecting the leading and trailing edge is the chord line. The distance from the leading to the trailing edge measured along the chord line is the chord of the aerofoil. The thickness is the distance between the upper and lower surfaces, perpendicularly to the chord line. The angle of attack is defined as the angle between the relative wind and the chord line [5].

The design of an aerofoil cross-section is governed by parametric equations based on form generation standards. 
The cross-sections are created by fitting spline curves through points generated from equations. The in-house developed programs, scripts, are used to generate point's coordinates on the specified radius of the blade, create splines and finally generate the loft surfaces and the body. To input every single point by coordinates for every crosssection is too tedious and error prone work. The modifications are even more difficult to implement.

Prediction of the aero-dynamical loads on a wind turbine requires a thorough understanding of the rather complex flow fields around wind turbine blades. The presence of three-dimensional effects makes modelling and prediction of these flow fields highly complicated.

Several methods are developed to assess the performance of wind turbines. BEM has been used for initial studies and coarse design due to simplicity. The method is a one dimensional approach dividing the blade into annular elements and therefore neglecting all 3D effects [6]. The simplified calculations introduce significant errors and introduction of correction factors has only showed modest improvement of the results. CFD software utilizes numerical methods for solving complex $3 \mathrm{D}$ problems such as motion of flows around wind turbine blades. CFD methods that take 3D effects into account are much more computational demanding.

\subsection{Creating blade geometry}

We implement the application in MS Excel for a generic model of a wind turbine blade [7]. Macro programming is one of several automation techniques that are usually included within Computer-Aided Design (CAD) systems. Macros are scripted instructions that are executed in interpretive way by the process running simultaneously within CAD system. Many CAD systems have supplemented their original macro programming languages with VBA; consequently, the main advantage of using VBA is utilization of integration and simplification with other applications based on the Windows framework. Macro programming provides the possibility of executing the same operations as could be performed by the graphical

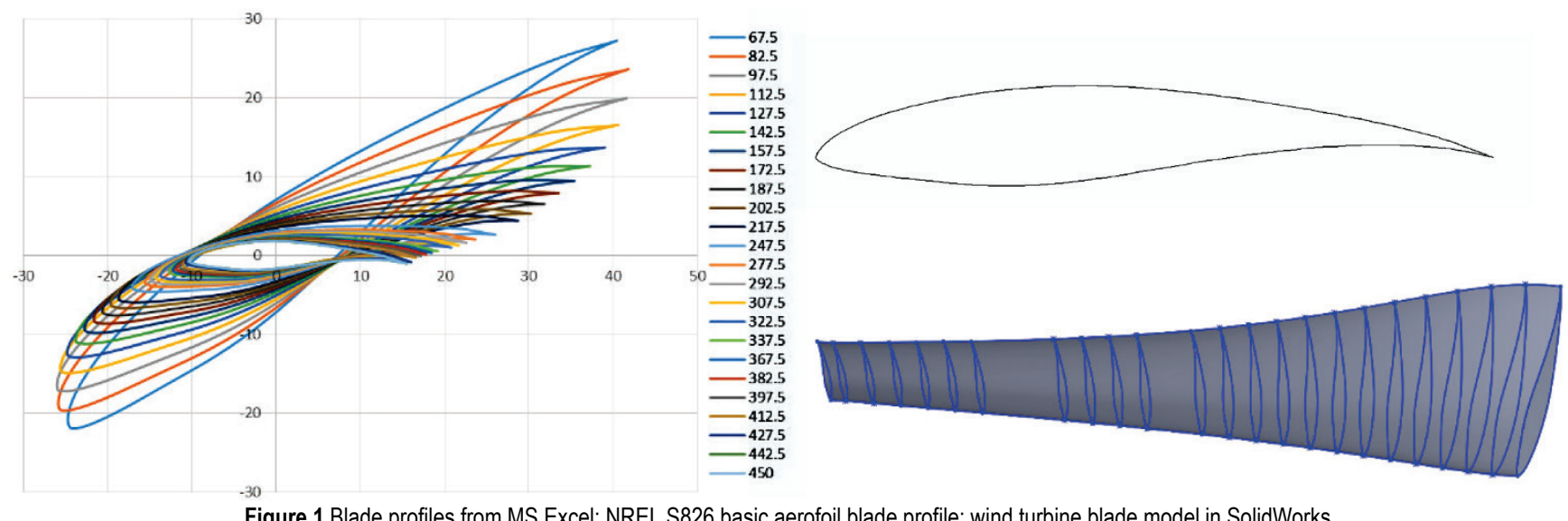

user interface. Macros execute tasks slower than compiled code but are still faster and more error free than manual work.

The generic model [7] that is developed in this regard is able to automate the process of the creation and modification of the wind blade geometry, based on a series of parameters that define the geometrical characteristics of a wind blade. The CAD automation is useful when the modelling tasks are practically impossible to be done manually because the very large number of points and great effort needed to create such geometry is time consuming, error prone and very precise and delicate work. Automation is welcome when there are more users who need to repeatedly reuse variants of the same components and when the automation process reduces the time needed for managing large numbers of models or models with such complex geometry with large number of calculated points.

The wind blade was designed using the NREL S826 aerofoil, the diameter of the wind turbine was $0.9 \mathrm{~m}$, rotation of $1300 \mathrm{rpm}$ and wind speed of $10 \mathrm{~m} / \mathrm{s}$. The blade was divided into sections, of $15 \mathrm{~mm}$, along the span-wise direction from the tip to the root. The BEM design method was used to calculate the chord length and twist angle of every blade element. For calculation of the blade aerodynamic forces the well-publicised BEM theory is applied, as [8] on the small wind turbines. Working along the blade radius taking small element, the sum of the aerodynamic forces can be calculated to give the overall blade reaction and thrust loads.

We used the in-house application to calculate profiles, rotate them according to their centre of gravity to get threedimensional coordinates of each blade section. For each section in span-wise direction the coordinates were calculated at a distance of $15 \mathrm{~mm}$, and the relations between the torsion angles of the blade, as can be seen in Fig. 1 in the axial view. We used macro-program to generate a solid body of the blade in SolidWorks 2017. The chords of each blade section along the span-wise direction are seen on the CAD model (Fig. 1 right).

From SolidWorks we exported the model to ANSYS 17.1 via STEP standard file.

\section{NUMERICAL DOMAIN AND BOUNDARY CONDITIONS}

For the CFD analysis SolidWorks 2017 Flow and ANSYS 17.1 CFX were used. The numerical domain was large enough ( $4 \mathrm{~m}$ before and $7 \mathrm{~m}$ behind the blade) to reduce the impact of domain walls to the geometry analysis. The size of the domain was specified the same for both software packages (Fig. 2). Steady state simulation was performed for both software packages, the Velocity Inlet was given as a boundary condition, of $10 \mathrm{~m} / \mathrm{s}$ on one side of the domain; on the other side of the domain, we used the Pressure Outlet boundary condition, where a 
pressure outlet without gauge pressure $(0 \mathrm{~Pa})$ was set. The left and right surfaces were defined as rotational periodicity. The upper surface and blade surface were defined as no slip walls. Air density during the analysis was $1.225 \mathrm{~kg} / \mathrm{m}^{3}$, dynamic viscosity $1.79 \times 10^{-5} \mathrm{~kg} /(\mathrm{m} \mathrm{s})$. The analysis was performed for an incompressible fluid and isothermal conditions. In ANSYS we have an option to select between different turbulence models, we selected the Shear-Stress-Transport (SST) turbulence model, which is commonly used for simulations of this type. SolidWorks does not provide the options for the turbulence model, so we are limited to the default k- $\varepsilon$ turbulence model.
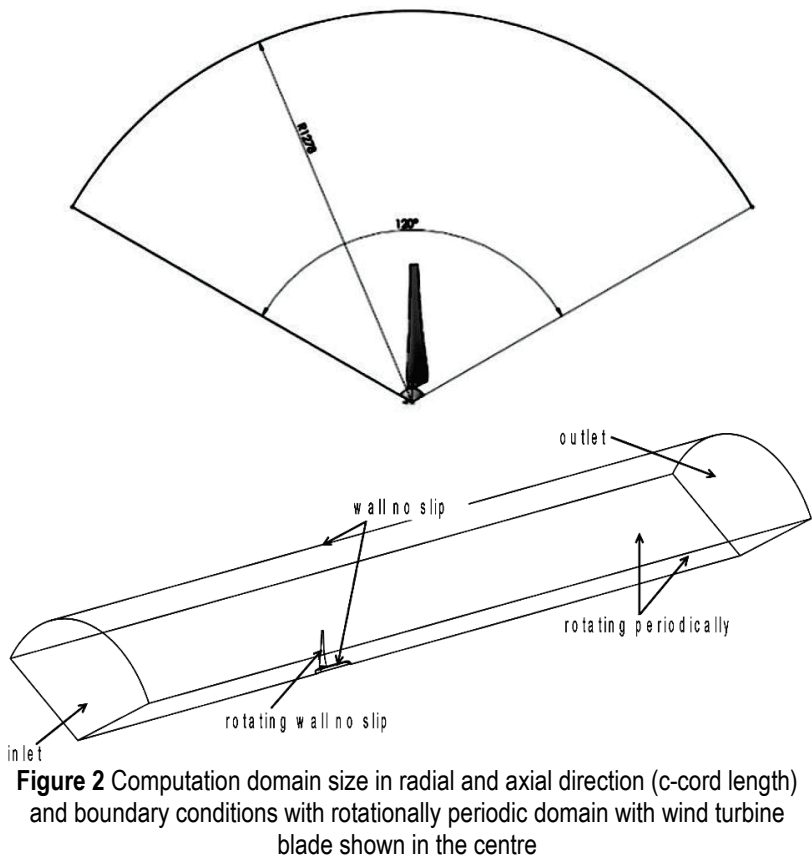

\subsection{Numerical Mode}

In order to create the computational domain and generate the mesh, the inbuilt software for meshing was used (SolidWorks Flow Simulation 2017 and ANSYS ICEM CFD 17.1). SolidWorks Flow Simulation uses a Cartesian based mesh, which has its advantages in simplicity, speed and robustness of the mesh generation algorithm (Fig. 3). Using the software tools, an additional meshing of the volume was performed around the body with local mesh sizing. The disadvantage of this meshing type is in that it gives the user very limited tools to sufficiently resolve the boundary layer.

In ANSYS a blocked structured mesh was created, with a first layer thickness of $0.02 \mathrm{~mm}$ and a growth ratio of 1.2. A level 2 refinement was used in the boundary layer in the axial direction. The advantages of creating a blocked structured mesh is in that it gives the user more freedom in designing a mesh. Compared to the Cartesian based meshing method used in SolidWorks it lets the user define the number of elements in $x, y, z$ directions and the growth ratios in all those direction. Refinement is not limited to all directions (which is the case for the Cartesian based mesh) but can be chosen in which direction it is performed. The above mentioned advantages result in a mesh that is sufficiently refined in areas near the blade surface and coarse in the areas where there are no huge pressure gradients. The disadvantage of the blocked structured mesh is that it takes more time and effort to create.
As shown in Fig. 3, one important part of the mesh shape is that it must be dense enough to be suitable for aerofoil shape. The number of elements for the CFD domain in SolidWorks was 6013016 elements and in ANSYS 2319208 elements.

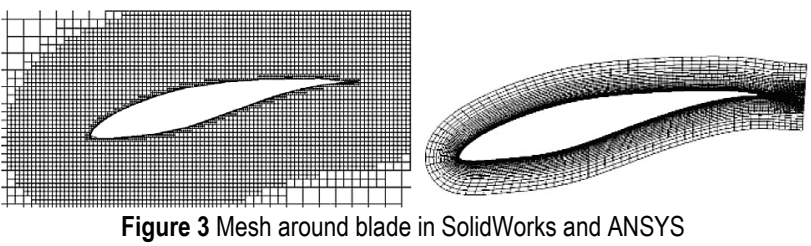

Analyses in each software were performed in two steps.

1) CFD analysis to get load on blade. Within this analysis we perform two situations a) with no prescribed rotation of blade, so no centrifugal load and b) with rotation of $1300 \mathrm{rpm}$ of the blade where maximal torque was expected from design calculations.

2) Structural analysis of the blade with transferring the results of CFD analysis as load on blade from both CFD analysis.

For the structural simulations the mesh in SolidWorks was created in SolidWorks Simulation 2017. The created mesh was unstructured and consisted of 67119 elements. In ANSYS the mesh was created using MECHANICAL 17.1. The created mesh was unstructured and consisted of 52040 elements.

For the structural analysis the parameters of the blade are shown in Tab. 1.

Table 1 Material parameters of ABS Tough plastics

\begin{tabular}{|c|c|c|c|}
\hline $\begin{array}{c}\text { Mass density } \\
\mathrm{kg} / \mathrm{m}^{3}\end{array}$ & $\begin{array}{c}\text { Poisson's } \\
\text { ratio }\end{array}$ & $\begin{array}{c}\text { Elastic modulus } \\
\mathrm{GPa}\end{array}$ & $\begin{array}{c}\text { Yield strength } \\
\mathrm{GPa}\end{array}$ \\
\hline 1215 & 0.42 & 2.50 & 1.80 \\
\hline
\end{tabular}

\section{ANALYSIS RESULTS}

Within SolidWorks, we take advantage of an integrated feature which allows to export Flow Simulation loads into Finite Element Analysis (FEA) studies, so results from CFD analysis were used as a loading condition in SolidWorks Simulation. The similar procedure could be performed in ANSYS.

\subsection{CFD Analysis Results}

The CFD simulations were performed in SolidWorks 2017 Flow Simulation and in ANSYS 17.1 CFX with different loads (blade rotation). In Fig. 4 are presented results from CFD analysis from SolidWorks and ANSYS in axial and radial directions at half of the length of the blade with no rotation of the blade. In Fig. 5 the results from both software for rotating blade are shown. The results are presented at the same scale for easier comparison. We could observe quite similar results for the non-rotating blade (cantilever beam with complex crosssection). The results for the rotating blade differ a lot that could be seen from velocity field. From ANSYS results the rotations are clearly seen as well as flow separation at blade. The differences in result are more pronounced in structural analysis. 

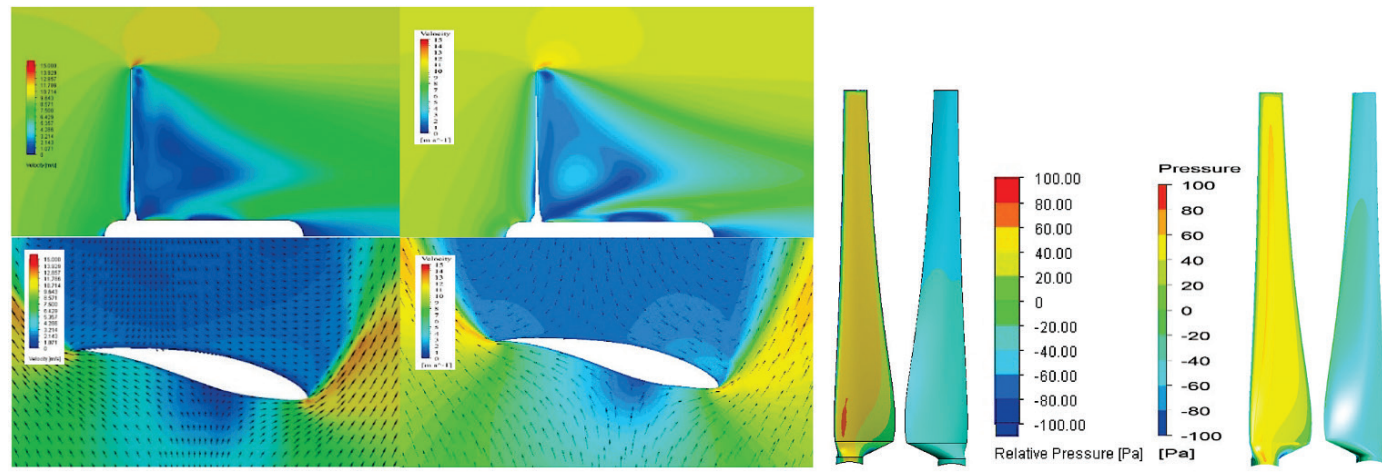

Figure 4 CFD results from SolidWorks and ANSYS with no rotation of the blade; velocities in axial and radial direction at 0.5R; pressure distribution on blade both sides
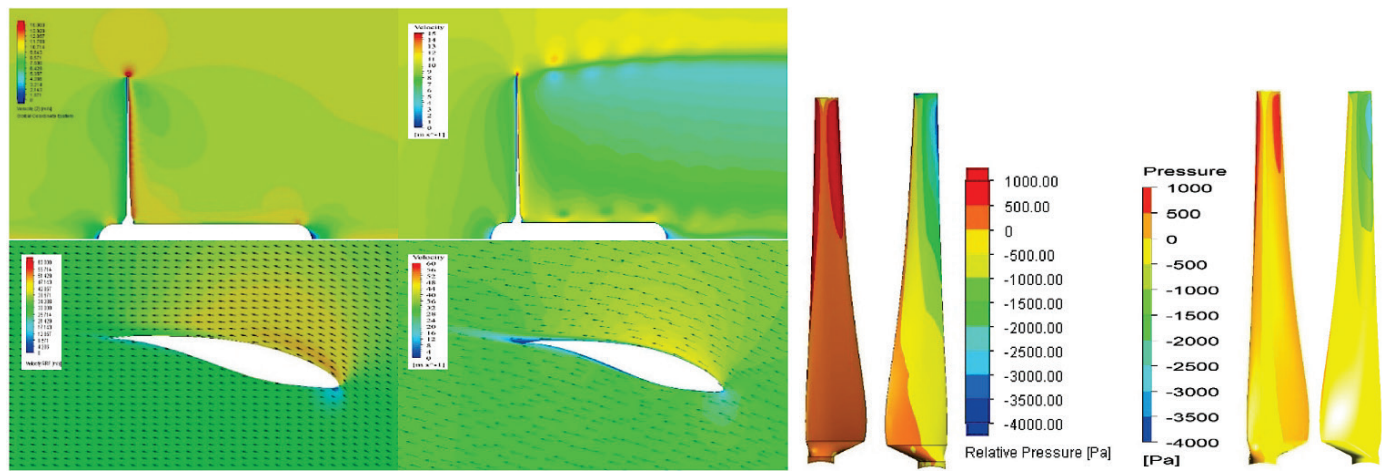

Figure 5 CFD results from SolidWorks and ANSYS with blade rotation; velocities in axial and radial direction at $0.5 \mathrm{R}$; pressure distribution on blade both sides

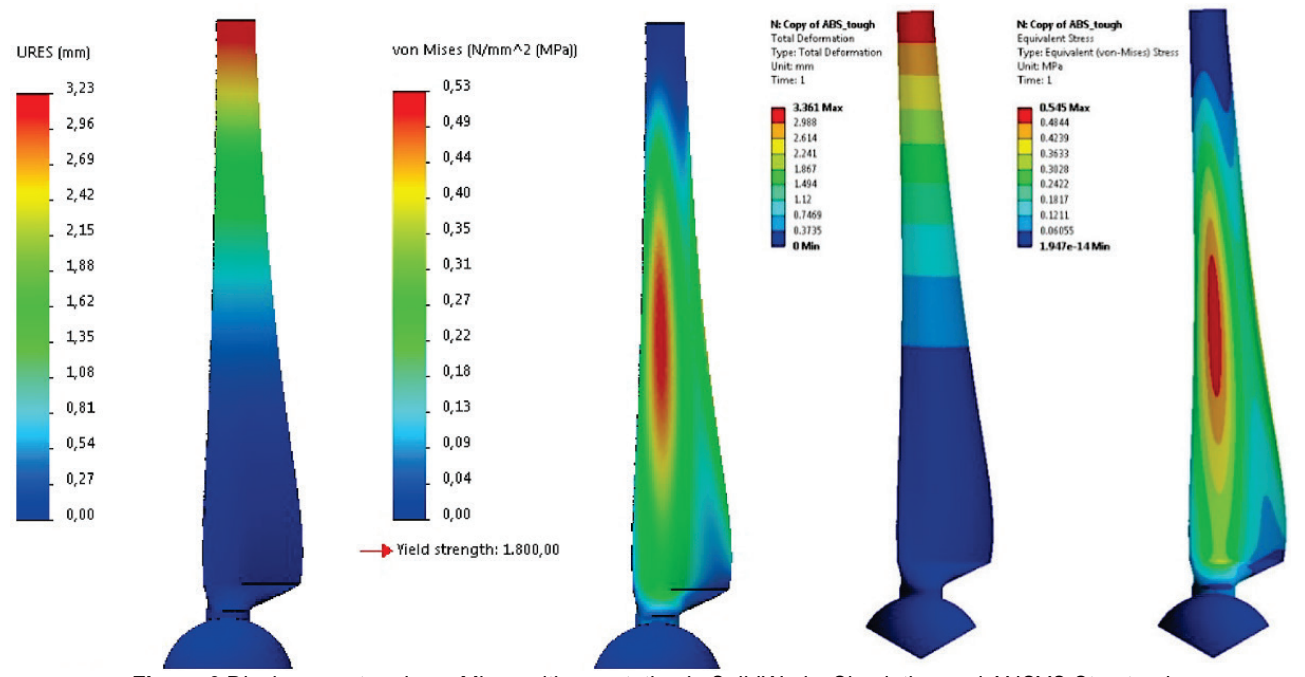

Figure 6 Displacement and von-Mises with no rotation in SolidWorks Simulation and ANSYS Structural

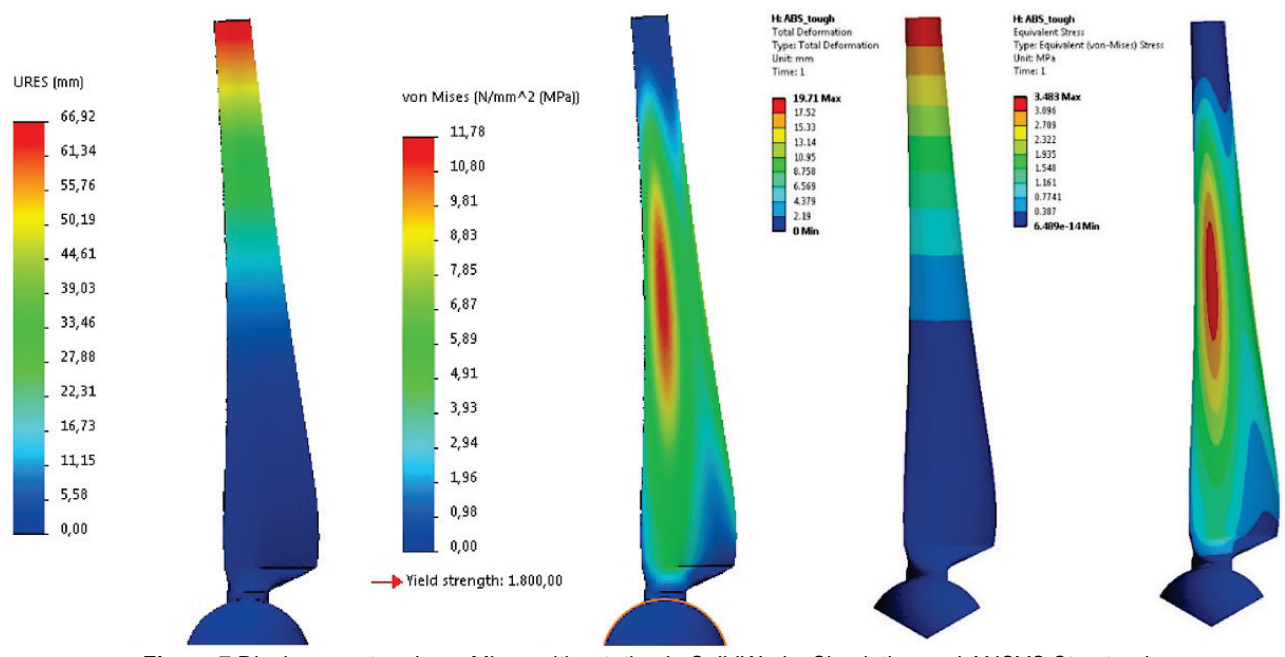

Figure 7 Displacement and von-Mises with rotation in SolidWorks Simulation and ANSYS Structural 


\subsection{Structural Analysis Results}

The results, pressure distribution on the blade surface were transferred for both cases for both software systems. The results obtained from simulations differ a lot, although the displacement and stresses (von Mises) distribution are adequate.

The displacement and stress distribution in the blade under the wind load only, were obtained from both software. Fig. 6 shows results of displacement and distribution diagram of von Mises stress of the blade. The picture illustrates that there is a stress concentration near the central of the blade. Obviously, the middle of the blade is the most easily damaged place when it is working under the aerodynamic loads, the second is the root of the blade. The maximum stress appears in the central position of the blade, about $0.53 \mathrm{MPa}$, that is much lower than the yield stress of the material. The displacements and von Mises stresses from ANSYS are showing minimal deviation.

In Fig. 7 the difference in pressure distribution for a rotating blade is clearly seen and that is used as a load for the structural simulations. Obtained results are presented in Fig. 7, where there can be seen that the distribution of displacement and stresses is as expected, but values of displacements and stresses are incomparable. SolidWorks is giving maximal displacement of $66.92 \mathrm{~mm}$ and maximal von Mises stress of $11.78 \mathrm{MPa}$ and ANSYS maximal displacement of $19.71 \mathrm{~mm}$ and maximal von Mises stress of $3.48 \mathrm{MPa}$.

\section{DISCUSSION AND CONCLUSION}

The main purpose of this work is to design an experimental size wind turbine and perform the whole design cycle, from design, simulations, manufacturing and measurements. The complex geometry of the aerofoil blade profile in this size could be produced with adding material technology, 3D printing. The design was carried out by BEM theory in a developed application to transfer all geometry data into the CAD system and automatically generate a wind turbine blade as a solid body. CFD module was engaged to gain loads on the blade, which was transferred into FEM module as a pressure distribution load. The displacements and von Mises stresses were calculated for material used for $3 \mathrm{D}$ printing. The simulations were performed in CAE specialised software in order to confirm results. The results are found to be understandable equal if we put the blade in space with wind as only load. According to the displacement solution of the blade, the maximal displacement occurs at the blade tip and the stress concentration occurs in the centre of the blade, which corresponds to the comprehensive stress from the elastic stress solution of the wind turbine blade. If we consider rotation of the blades, the results are showing that integrated software gives much different and less reliable results.

The comparison between integrated software, SolidWorks, and specialised software for numerical analysis, ANSYS, for other less complex examples [9-11] showed quite good consistency of results.

The performed comparison analysis of the prediction of numerical analysis with the ANSYS software package, and the SolidWorks software package shows a difference between the predictions of flow field and values. The SolidWorks software package is significantly more inaccurate in prediction of results, and needs significantly more time. The difference between the predictions among different packages can be explored in the meshing technique as well as in the used numerical algorithms. The performed analysis, both from the aspect of results accuracy as well as from that of computational times, shows that purpose-made tools for numerical analysis, such as ANSYS, are much faster and more accurate than add-ons to 3D modellers, as in the case of the SolidWorks.

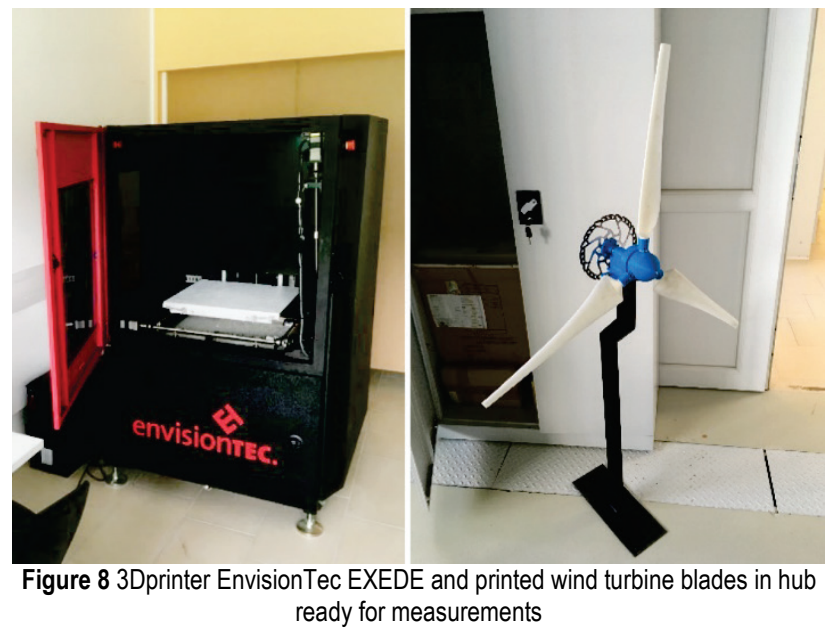

It could be concluded integrated software is appropriate for simulations that are "simple" enough in early stages of design, but useless in more complex physical phenomena description. Appropriate creation of numerical mesh, boundary conditions to prescribe physical conditions and solver capability are key issues to perform simulations with reliable results.

We are working on CFD simulations for other angular velocities and experimental work to confirm simulation results with measurements (Fig. 8) and prove our assumptions.

\section{REFERENCES}

[1] Beckers, R. (2018). The Truth About Small Wind Turbines, Solacity Inc., https://www.solacity.com/small-wind-turbinetruth/ (7.4.2018) https://doi.org/10.1515/9781614514176-002

[2] Burton, T. D., Sharpe, D., Jenkins, N., \& Bossanyi, E. (2001). Wind Energy Handbook (1 ed.), West Sussex: John Wiley \& Sons. https://doi.org/10.1002/0470846062

[3] Ding, J., Wang, H., Ping, L. S., \& Ma, B. (2013). Optimal Design of Wind Turbine Blades with Wilson and BEM Method Integrated. Applied Mechanics and Materials, (ed. Zhang J., Honghua T.), 404, 286-291. https://doi.org/10.4028/www.scientific.net/AMM.404.286

[4] Sagmo, K. F., Bartl, L., \& Sætran, T. (2016). Numerical simulations of the NREL S826 aerofoil. Journal of Physics: Conference Series, 735082036. https://doi.org/10.1088/1742-6596/753/8/082036

[5] Spera, D. A. (2009). Wind turbine technology: Fundamental concepts of wind turbine engineering (2 ed.). https://doi.org/10.1115/1.802601

[6] Wang, H., Ma, B., Ding, J., \& Li, S. (2014). The Modeling and Stress Analysis of Wind Turbine Blade. Indonesian Journal of Electrical Engineering, 12(6), 4178-4183. https://doi.org/10.11591/telkomnika.v12i6.5536 
[7] Hren, G., Predin, A., \& Žagar, I. (2013). Generic model of wind turbine blades. Journal of Energy Technology, 6, 6168.

[8] Khalil, Y., Tenghiri, L., Abdi, F., \& Bentamy, A. (2017). Efficiency of a small wind turbine using BEM and CFD. IOP Conference Series: Earth and Environmental Science, (REEE'2017), Volume 161. https://doi.org/10.1088/1755-1315/161/1/012028

[9] Ščuri I., Pezdevšek, M., Spaseski, I., Fike, M., \& Hren, G. (2014). Numerical analysis of an axial flow fan: ANSYS vs SolidWorks. Journal of Energy Technology, 4, 11-19.

[10] Spaseski, I., Pezdevšek, M., Ščuri I., Fike M., \& Hren, G. (2014). Numerical analysis of lift and pressure coefficients of an aerofoil: Ansys vs SolidWorks. Journal of Energy Technology, 7, 63-73.

[11] Pezdevšek, M., Spaseski, I., Ščuri I., Fike M., \& Hren, G. (2015). Numerical analysis of a heat exchanger: Ansys vs SolidWorks. Journal of Energy Technology, 8, 51-62.

\section{Contact information:}

Gorazd HREN, Dr. -Ing., Assistant Professor

University of Maribor

Faculty of Energy Technology,

Hočevarjevtrg 1

8270 Krško, Slovenia

gorazd.hren@um.si 